\title{
Structure, Dynamics, and Composition of Large Clusters in Polyelectrolyte-Surfactant Systems
}

\author{
Ingo Hoffmann, ${ }^{*}{ }^{\dagger, \ddagger \odot}$ Miriam Simon, ${ }^{\ddagger}$ Markus Bleuel, ${ }^{\S, \|}$ Peter Falus, ${ }^{\dagger}$ and Michael Gradzielski*, ${ }^{\ddagger}$ \\ ${ }^{\dagger}$ Institut Max von Laue-Paul Langevin (ILL), 71 avenue des Martyrs, CS 20156, Cedex 9, F-38042 Grenoble, France \\ ${ }^{\ddagger}$ Stranski-Laboratorium für Physikalische und Theoretische Chemie, Institut für Chemie, Technische Universität Berlin, Straße des \\ 17. Juni 124, Sekr. TC 7, D-10623 Berlin, Germany \\ ${ }^{\S}$ NIST Center for Neutron Research, National Institute of Standards and Technology, 100 Bureau Drive, Gaithersburg, Maryland \\ 20899-6102, United States \\ "Department of Materials Science and Engineering, University of Maryland, College Park, Maryland 20742-2115, United States
}

Supporting Information

ABSTRACT: Mixtures of oppositely charged polyelectrolyte (PE) and surfactant show rich structural behavior. The dynamics of such self-assembled structures can be quite complex. For example, using dynamic light scattering (DLS), we observed a slow collective relaxation mode for mixtures of the cationically charged PE JR 400 and the anionic surfactant SDS if the concentration of the system is sufficiently high and a small excess of PE charges is present. A similar mode is observed by using fluorescence correlation spectroscopy
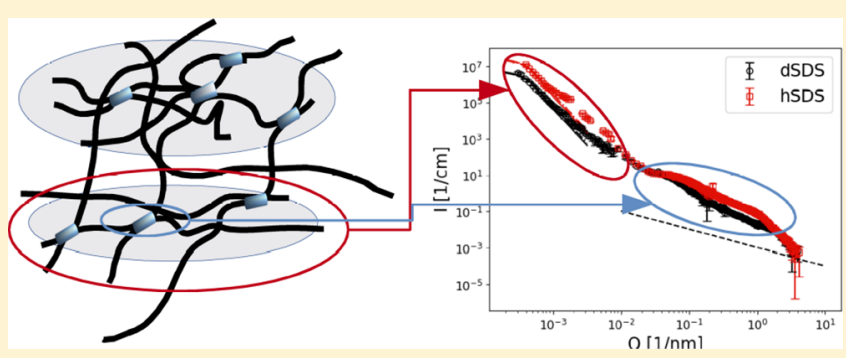
(FCS). However, a large quantitative discrepancy is observed between the values obtained from DLS and FCS. In this paper, we are investigating the connection between these slow relaxation modes and the formation of large micrometer sized structures which have been investigated using ultrasmall-angle neutron scattering (USANS), and the discrepancies between results from DLS and FCS are explained.

\section{INTRODUCTION}

Mixtures of oppositely charged polyelectrolytes (PEs) and surfactants show rich self-aggregation behavior and can be found in formulations for detergency, ${ }^{1}$ oil recovery, ${ }^{2}$ and cosmetics $^{3}$ and therefore have attracted much interest over the past decades. $^{4-23}$

Depending on the composition of the sample, the charge density and backbone rigidity of the $\mathrm{PE}$ and the packing parameter of the surfactant different shapes of aggregates are observed. $^{24-26}$ For example, pearl-necklace structures, rodlike aggregates, or lamellar structures can be observed in polyelectrolyte-surfactant complexes (PESCs).

Some of these PESCs show a remarkable increase in viscosity, while others have only very little influence on the rheological behavior of the system. In particular, mixtures of several anionic surfactants and the cationically modified hydroxyethyl cellulose JR 400 greatly increase the viscosity of solutions, ${ }^{27}$ while other quite similar systems have only very little effect. An example for such a situation is hyaluronate, where the addition of oppositely charged surfactant has basically no effect on the viscosity. ${ }^{28}$ In contrast, for the addition of dodecyltrimethylammonium bromide (DTAB) to poly(acrylic acid) (PAA) a substantial decrease of the viscosity upon DTAB addition has been reported. ${ }^{29}$

Of course, of central importance for the understanding of the properties of self-assembled systems is the mesoscopic structure. ${ }^{30}$ In some of the systems, where no large impact on viscosity is observed, PESC superstructures are formed in the form of clusters of PE/surfactant aggregates ${ }^{28,31-35}$ with sizes on the order of a few $100 \mathrm{~nm}$, while in SDS/JR 400 and SDBS/JR 400 scattering from large clusters beyond the range accessible by small-angle neutron scattering (SANS) is observed. In addition to a faster relaxation related to the size of the primary aggregates, a slow mode is seen in DLS which is related to the relaxation of the clusters. ${ }^{36-39}$ In general, one may speculate that this slow mode seen in PESCs might be related to that observed for pure polyelectrolyte solutions where also the presence of large domains/inhomogeneities has been claimed. ${ }^{40-42}$

In previous publications we performed SANS and NSE measurements on highly viscous SDS/JR 400 mixtures and observed the formation of rodlike aggregates with a length of about $50 \mathrm{~nm},{ }^{43,44}$ by applying the Broersma equation for the diffusion of rodlike particles. ${ }^{45}$ As opposed to the radius, the length of the rodlike aggregates could not be simply obtained from SANS, as the scattering from the rods is buried under the scattering from larger clusters. It was found that the rods interconnect multiple JR 400 chains, thereby causing the

Received: December 7, 2018

Revised: February 25, 2019

Published: March 15, 2019 
formation of large clusters, which explains the increase of the viscosity of the solution.

In the present paper we investigate these large clusters apparently formed in JR 400/SDS mixtures and their relaxation in detail as their larger size in comparison to other systems where clusters of only a few $100 \mathrm{~nm}$ were observed might be related to the increased viscosity observed in mixtures of JR 400 and anionic surfactants. For that purpose we combined a variety of dynamic methods like neutron spin-echo (NSE) spectroscopy, dynamic light scattering (DLS), and fluorescence correlation sprectroscopy (FCS) with the corresponding static methods, such ultrasmall-angle neutron scattering (USANS) and SANS.

\section{MATERIALS AND METHODS}

Methods. Small-angle neutron scattering (SANS) measurements were performed using the NG7SANS instrument (NCNR, NIST, Gaithersburg, $\mathrm{MD})$. The $Q$-range $(Q=4 \pi / \lambda \sin (\theta / 2)$, where $\theta$ is the scattering angle) of $1 \times 10^{-2}$ to $51 / \mathrm{nm}$ was achieved using three standard detector geometries ( $1 \mathrm{~m}$ with an $0.25 \mathrm{~m}$ detector offset, 4 $\mathrm{m}$, and $13 \mathrm{~m}$ ) with $6 \AA$ neutrons and the neutron lens setting at 8.13 $\AA$ neutron wavelength. The samples were housed in $5 \mathrm{~mm}$ standard cell holders with quartz windows and measured for $60 \mathrm{~min}$. Scattering data were normalized for the sample transmission and background corrected using a quartz cell with $\mathrm{D}_{2} \mathrm{O}$ and also corrected for the linearity and efficiency of the detector response using the NCNR Igor macros. $^{46}$

Ultrasmall-angle neutron scattering (USANS) measurements of the samples were performed using the BT5 instrument (NIST Center for Neutron Research (NCNR), National Institute for Standards and Technology (NIST), Gaithersburg, MD). A Q-range of $3 \times 10^{-4}$ to $0.001 .1 \times 10^{-2} 1 / \mathrm{nm}$ was achieved using a Bonse-Hart type double crystal diffractometer with a wavelength $(\lambda)$ of $2.4 \AA(\Delta \lambda / \lambda=6 \%)$ in the standard geometry. ${ }^{46}$ Samples were housed in $5 \mathrm{~mm}$ quartz cuvettes and measured for $4 \mathrm{~h}$ each. Scattering data were normalized for the sample transmission and background corrected using a quartz cell with $20 \% \mathrm{v} / \mathrm{v} d_{4}$-methanol in $\mathrm{D}_{2} \mathrm{O} .^{47}$

The scattering intensity of polydisperse noninteracting objects is given by

$$
I(Q)={ }^{1} N \int_{0}^{\infty} f(R) F(Q, R)^{2} \mathrm{~d} R+I_{\mathrm{bkg}}
$$

where $f(R)$ is a distribution function, $F(Q R)$ is the scattering amplitude of the objects (where the $F^{2}$ is usually termed the form factor $P), R$ is the size which is subject to the distribution, and $I_{\mathrm{bkg}}$ is the incoherent background. The relation between the particle number density ${ }^{1} N$, the volume fraction of the objects $\phi$, the size distribution function $f(R)$, and the shape of the objects is given by

$$
{ }^{1} N=\frac{\phi}{\int_{0}^{\infty} f(R) V(R) \mathrm{d} R}
$$

where $V(R)$ is the volume of the objects with size $R$.

Here, we used the normalized log-normal distribution function:

$$
f\left(R, R_{\mathrm{m}}, \sigma\right)=\frac{1}{\sqrt{2 \pi} \sigma R} \exp \left(-\frac{\ln \left(R / R_{\mathrm{m}}\right)^{2}}{2 \sigma^{2}}\right)
$$

where the moments of the distribution $\left\langle R^{(n)}\right\rangle$ are

$$
\left\langle R^{(n)}\right\rangle=R_{\mathrm{m}}{ }^{n} \exp \left(n^{2} \sigma^{2} / 2\right)
$$

so that the mean value of the distribution is given by $\left\langle R^{(1)}\right\rangle=R_{\mathrm{m}}$ $\exp \left(\sigma^{2} / 2\right)$ and the standard deviation is given by $\sqrt{\exp \left(\sigma^{2}\right)-1} R_{\mathrm{m}} \exp \left(\sigma^{2} / 2\right)$.

The scattering amplitude of a sphere is given by

$$
F(Q, R, \Delta \mathrm{SLD})=\frac{4 \pi}{3} R^{3} \Delta \mathrm{SLD}\left(3 \frac{\sin (Q R)-Q R \cos (Q R)}{(Q R)^{3}}\right)
$$

where $\Delta$ SLD denotes the difference in scattering length density SLD between the object and the matrix.

For $Q \gg 1 / R$, irrespective of the shape of the object for a sharp interface a decay with slope $Q^{-4}$ is observed, and the proportionality constant is called the Porod constant ${ }^{48}$ and related to the surface of the objects $\langle S\rangle$ :

$$
B={ }^{1} N 2 \pi \Delta \operatorname{SLD}^{2}\langle S\rangle
$$

For polydisperse spheres, this becomes $B_{\mathrm{s}}={ }^{1} N 8 \pi^{2} \Delta \mathrm{SLD}^{2} \int_{0}^{\infty} f(R) R^{2}$ $\mathrm{d} R$. Inserting eq 2

$$
B_{\mathrm{s}}=6 \pi \phi \Delta \operatorname{SLD}^{2} \frac{\int_{0}^{\infty} f(R) R^{2} \mathrm{~d} R}{\int_{0}^{\infty} f(R) R^{3} \mathrm{~d} R}
$$

From eqs 6 and 7 it can be seen that the scattering intensity at a given $Q$ decreases as the objects become larger since the surface per volume decreases and the intensity is proportional to $\frac{\left\langle R^{(2)}\right\rangle}{\left\langle R^{(3)}\right\rangle}$ which reduces to $1 / R$ in the monodisperse case. ${ }^{49}$

Neutron spin-echo spectroscopy (NSE) experiments were performed on the instrument IN15 (ILL) at wavelengths of 10 and $16 \AA$, allowing to reach maximum Fourier times of 50 and $200 \mathrm{~ns}$, respectively. Details of the experiment, the method, and applications to soft matter samples are explained elsewhere. ${ }^{50-57}$ The method yields the intermediate scattering function $S(Q, t)$, which yields an apparent diffusion coefficient $D_{\text {app }}$ via

$$
S(Q, t) / S(Q, 0)=\exp \left(-D_{\text {app }} Q^{2} t\right)
$$

and $t$ is the Fourier time, given by

$$
t=\frac{\gamma_{\mathrm{N}} J m_{\mathrm{N}}^{2}}{2 \pi h^{2}} \lambda^{3}
$$

The gyromagnetic ratio of the neutron $\gamma_{\mathrm{N}}$, its mass $m_{\mathrm{N}}$, and the Planck constant $h$ are all constants, while the field integral $J$ and the wavelength of the neutrons $\lambda$ are changed in an experiment.

Dynamic light scattering (DLS) measures the intensity autocorrelation function $g^{(2)}$, which is related to the field autocorrelation function $g^{(1)}$ by the Siegert relation ${ }^{58}$

$$
g^{(2)}=A\left|g^{(1)}\right|^{2}+1
$$

and the collective diffusion coefficient $D_{c}$ is obtained from $g^{(1)}$

$$
g^{(1)}=\exp \left(-D_{c} q^{2} t\right)
$$

$D_{\mathrm{c}}$ can be approximated as ${ }^{59}$

$$
D_{\mathrm{c}}=\frac{D_{\mathrm{s}}}{S(Q)}
$$

where $D_{\mathrm{s}}$ is the self-diffusion coefficient and $S(Q)$ is the static structure factor so that for noninteracting particles $D_{\mathrm{c}}$ and $D_{\mathrm{s}}$ coincide.

A distribution of relaxation rates results in a broader decay and can be described with a stretched exponential

$$
g^{(1)}=\exp \left(-(t / \tau)^{\alpha}\right)
$$

with the stretch exponent $\alpha<1$. The $n$th moment of the distribution $\left\langle\tau^{(n)}\right\rangle$ is given by

$$
\left\langle\tau^{(n)}\right\rangle=\frac{\tau^{n}}{\alpha} \Gamma\left(\frac{n}{\alpha}\right)
$$

with the Gamma function $\Gamma(x)$ so that the mean relaxation time (first moment) is given by 


$$
\tau_{\mathrm{av}}=\frac{\tau}{\alpha} \Gamma\left(\frac{1}{\alpha}\right)
$$

and the resulting diffusion coefficient is $D_{\mathrm{av}}=\frac{1}{\tau_{\mathrm{av}} q^{2}}$.

The diffusion coefficient $D$ is related to the hydrodynamic radius $R_{\mathrm{H}}$ by the Stokes-Einstein equation:

$$
D=\frac{k_{\mathrm{B}} T}{6 \pi \eta R_{\mathrm{H}}}
$$

Measurements were performed on a setup with an ALV CGS 3 goniometer, an $\mathrm{ALV} 7004$ correlato, $\mathrm{r}$ and a $\mathrm{He}-\mathrm{Ne}$ laser with a wavelength of $632.8 \mathrm{~nm}$.

Fluorescence correlation spectroscopy (FCS) measurements were performed on a Leica TCS SP5 II confocal microscope with a TCS SP5 SMD single molecule detection unit. An argon laser $(\lambda=488$ $\mathrm{nm}$ ) was used for the excitation of Nile Red. Light was delivered to the sample through an apochromatic $63 \times, 1.2 \mathrm{NA}$ water immersion objective; the fluorescence light was collected through the same objective. Data were detected in pseudo-cross-correlation mode with a 50:50 beam splitter. The size of the confocal volume and its anisotropy have been determined before, using the dye Alexa48 8 with a known diffusion coefficient of $43.5 \AA^{2} / \mathrm{ns}^{60}$ The correlation functions were obtained on a Picoharp 300 correlator and could be described with the following expression, which has proven useful for the description of anomalous diffusion or the diffusion of polydisperse samples: ${ }^{61-63}$

$$
G(t)=\frac{1}{N}\left(1+\left(\frac{t}{\tau}\right)^{\alpha}\right)^{-1}\left(1+\left(\frac{t}{\tau k^{2}}\right)^{\alpha}\right)^{-0.5}
$$

$N$ is directly related to the number of dye molecules in the confocal volume, $\tau$ is the relaxation time and related to the apparent diffusion coefficient by $D_{\text {app }}=\frac{\omega_{x, y}{ }^{2}}{4 \tau}$, and $\omega_{x, y}$ is the radius of the confocal volume along the $x$-and $y$-axis, while the $z$-axis is elongated by a factor $k$, so that $\omega_{z}=\omega_{x, y} k$. The shape of the confocal volume is assumed to be Gaussian, so that the intensity along the $n$-axis is $I(n)=I_{0} \exp ((n)$ $\left.\left.2 \omega_{n}\right)^{2}\right)$, with the center of the confocal volume at $n=0$. The stretch parameter $\alpha$ is related to the polydispersity of the sample in analogy to the exponent in the stretched exponential function. For a monodisperse sample $\alpha=1$. As opposed to the stretched exponential function, no analytical solution exists for the average relaxation time; therefore, a numerical integration has to be performed to obtain an average value:

$$
\tau_{\mathrm{av}}=\frac{1}{c} \int_{0}^{\infty}\left(1+\left(\frac{t}{\tau}\right)^{\alpha}\right)^{-1}\left(1+\left(\frac{t}{\tau k^{2}}\right)^{\alpha}\right)^{-0.5} \mathrm{~d} t
$$

where $c$ is a constant such that $\tau_{\mathrm{av}}=1$ with $\alpha=1$ and $\tau=1$ and the average diffusion coefficient is $D_{\mathrm{av}}=\frac{\omega_{x, y}{ }^{2}}{4 \tau_{\mathrm{av}}}$.

Materials. JR 400 (Dow Chemical, USA) is a cationically modified hydroxyethylcellulose (cat-HEC) with a molecular weight of about $500000 \mathrm{~g} / \mathrm{mol}\left(\mathrm{PDI}=1.85,{ }^{64}\right.$ density in water $\left.1.66 \mathrm{~g} / \mathrm{mL}\right)$ and a cationic group on $27 \%$ of the glucose units, resulting in $1000 \mathrm{~g}$ of PE per mole of positive charges. ${ }^{65} h$-SDS (98.5\%) and $d$-SDS (99.4\% isotopic purity) were purchased from Sigma-Aldrich and MSD Isotopes, respectively, and used as received. Samples were simply prepared by dissolving the corresponding amounts of dry powder in $\mathrm{H}_{2} \mathrm{O}$ from a Millipore system, except solutions for neutron scattering experiments where $\mathrm{D}_{2} \mathrm{O}$ (Euriso-top, France) was used as solvent. The JR 400 concentration was adapted to account for the higher density of $\mathrm{D}_{2} \mathrm{O}$. Samples were prepared with both h-SDS and d-SDS, where $d$-SDS is matched by $\mathrm{D}_{2} \mathrm{O}$ and only the $\mathrm{PE}$ is visible, while with $h$-SDS both surfactant and PE are visible.

The concentration of SDS is given as charge ratio $Z=$ [polymer charges $] /[$ surfactant charges] or as molar concentration. The PE concentration was always 1 wt \% (which corresponds to $10 \mathrm{mM}$ charges). All samples are transparent with almost water-like turbidity (see Figure S1).

\section{RESULTS AND DISCUSSION}

The viscosity of mixtures of 1 wt \% JR 400 and SDS (and other anionic surfactants) with an excess of PE charges compared to the surfactant increases dramatically when adding more surfactant and thereby coming closer to the phase boundary. Around charge equilibrium the formation of a precipitate is observed and with an excess of surfactant the precipitate can be redissolved, but solutions with an excess of surfactant have a low viscosity (see Figure S2). We previously found that this is due to the formation of mixed rodlike aggregates which are about $2 \mathrm{~nm}$ in radius and about $50 \mathrm{~nm}$ long. ${ }^{43,44}$ They interconnect several PE chains and thereby act as cross-links between different PE chains. These structures are not present in an excess of surfactant, as they become simply dissolved. ${ }^{66}$ The length of the rodlike aggregates could not be determined from SANS as the onset of the plateau of the scattering from the rods, which would allow to determine their length, is hidden below the scattering from larger clusters. The length of the rods could only be determined using NSE, where a diffusion coefficient was obtained that could be attributed to the diffusion of the rodlike aggregates. With the radius known from SANS and using the Broersma equation ${ }^{45}$ for the diffusion of rods, the diffusion coefficient measured using NSE could be converted to a length, and a value on the order of 50 $\mathrm{nm}$ was obtained. See the Supporting Information section 1 for details about the calculation of the length of the rodlike aggregates from NSE data.

Here, we want to focus our attention on the structure and dynamics of the large clusters, which can be seen at low $Q$ and previously made it impossible to determine the length of the smaller aggregates from SANS. When performing NSE measurements using $16 \AA$ neutrons, which allow to access longer Fourier times, even in the NSE Q-range it could be observed that another slow contribution was starting to influence the dynamics and applying the Broersma equation would lead to apparently longer rodlike particles (see Figure 1). Therefore, we tried to describe the $16 \AA$ data with the fixed contribution from the $48 \mathrm{~nm}$ rods (previously obtained from

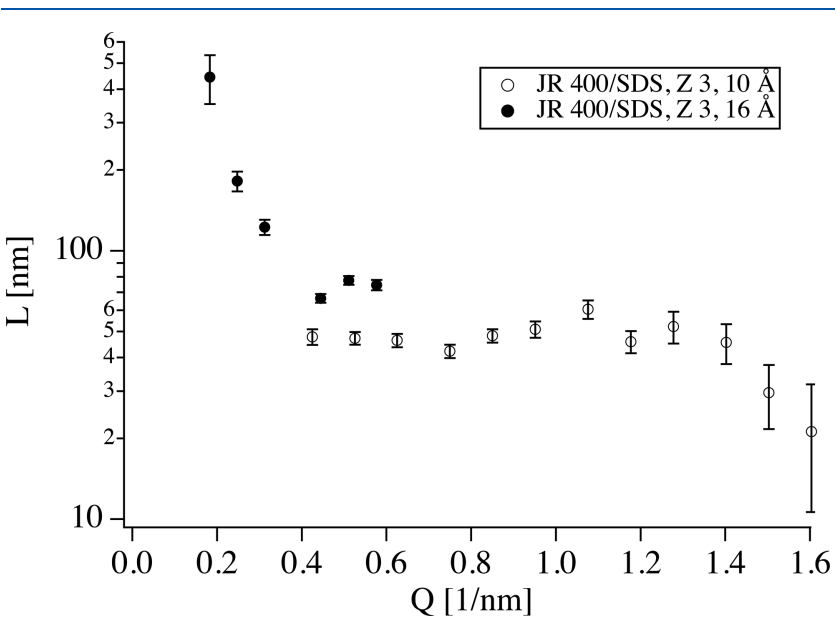

Figure 1. Length obtained from fitting NSE data ( $1 \mathrm{wt} \% \mathrm{JR} 400$ with $3.3 \mathrm{mM}$ SDS, $Z=3$ ) with the diffusion of rods; at long Fourier times $(\lambda=16 \AA)$ another slow contribution becomes visible, which is reflected in an apparent increase of the length. 
measurements with $10 \AA$ neutrons) and an additional slow diffusion coefficient according to

$$
S(Q, t)=A_{\text {fast }} S_{\text {rod }}(Q, t)+\left(1-A_{\text {fast }}\right) \exp \left(-D_{\text {slow }} Q^{2} t\right)
$$

However, it turns out that the time scale is too slow for NSE so that $D_{\text {slow }}$ was fixed at 0 , and only the amplitude of the fast contribution could be determined. Good fit results are obtained nevertheless (see Figure S3), and $A_{\text {fast }}$ is obtained as the only fit parameter (see Figure 2). It can be seen that the contribution of the fast mode decreases quickly toward low $Q$ and the slow mode from the large clusters becomes more important.

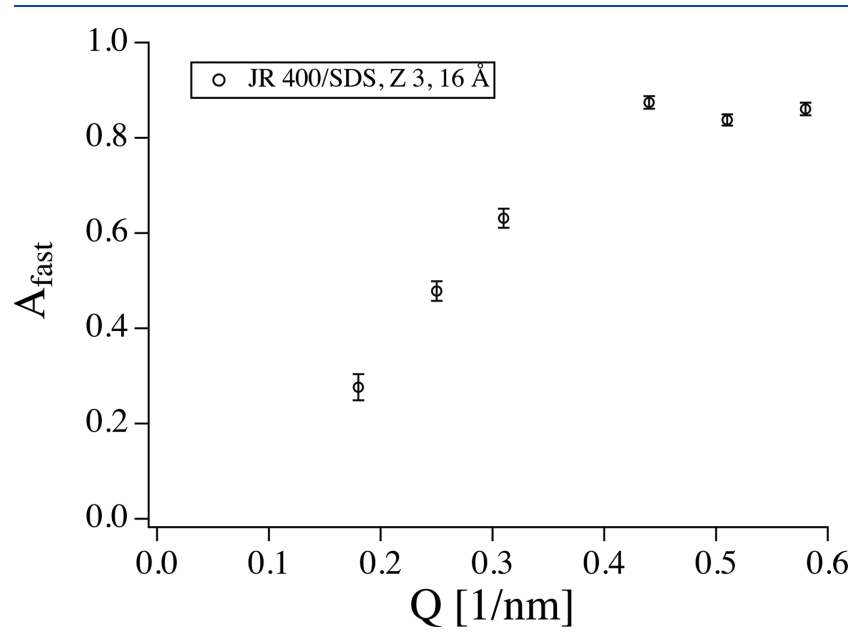

Figure 2. Relative contribution of the fast mode in NSE for $1 \mathrm{wt} \% \mathrm{JR}$ 400 with $3.3 \mathrm{mM}$ SDS $(Z=3)$ from fitting eq 19 as a function of $Q$. The relative contribution of the fast mode decreases with decreasing Q.

Therefore, we decided to perform DLS and FCS measurements which observe the dynamics at larger length scales and longer Fourier time.

FCS/DLS. FCS measurements using Nile Red as a hydrophobic label yielded correlation functions which can be nicely fitted using eq 17 (see Figure S4). The values of the stretch parameter $\alpha$ are close to 1 (see Figure S5), which indicates that there is no wide distribution of relaxation rates, but a single well-defined decay and the average diffusion coefficients obtained are on the order of $0.05 \AA^{2} / \mathrm{ns}$ (see Figure 3 ), corresponding to hydrodynamic radii on the order of 300 $\mathrm{nm}$. Only at the lowest SDS concentration a somewhat higher value for $D_{\text {av }}$ is obtained, and for all higher SDS concentrations $D_{\text {Av }}$ remains relatively constant.

The autocorrelation functions measured by DLS, on the other hand, clearly show a bimodal decay (see Figure 4) with a fast and a second much slower mode. The slow mode shows a distinctly nonexponential decay; therefore, data were described as a combination of an exponential and a stretched exponential:

$$
g^{2}(t)=f\left(A_{\text {fast }} \exp \left(-D_{1} Q^{2} t\right)+\left(1-A_{\text {fast }}\right) \exp \left(-\left(t / \tau_{2}\right)^{\alpha}\right)\right)^{2}
$$

where the slow diffusion coefficient $D_{2}$ is related to $\tau_{2}$ by $D_{2}=\frac{1}{\tau_{2} Q^{2}}$ and the average slow diffusion coefficient $D_{2 \mathrm{Av}}$ is obtained from the average relaxation time of the slow mode $\tau_{2 \mathrm{Av}}$ as given by eq 15 . This behavior is expected, has been

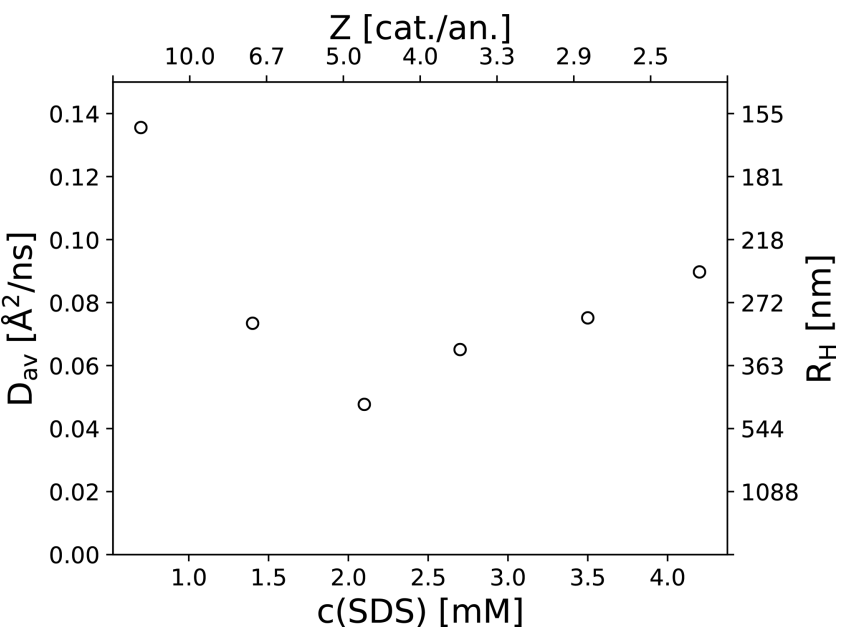

Figure 3. $D_{\text {av }}$ obtained from fitting FCS correlation functions with eq 17. All samples contained $1 \mathrm{wt} \% \mathrm{JR} 400$ and varying amounts of SDS. An initial decrease aside, no large changes in $D_{\mathrm{av}}$ are observed. The values obtained are significantly smaller than the values obtained for the fast mode in DLS and therefore do not correspond to the relaxation of the rodlike aggregates but are related to the relaxation of large clusters.

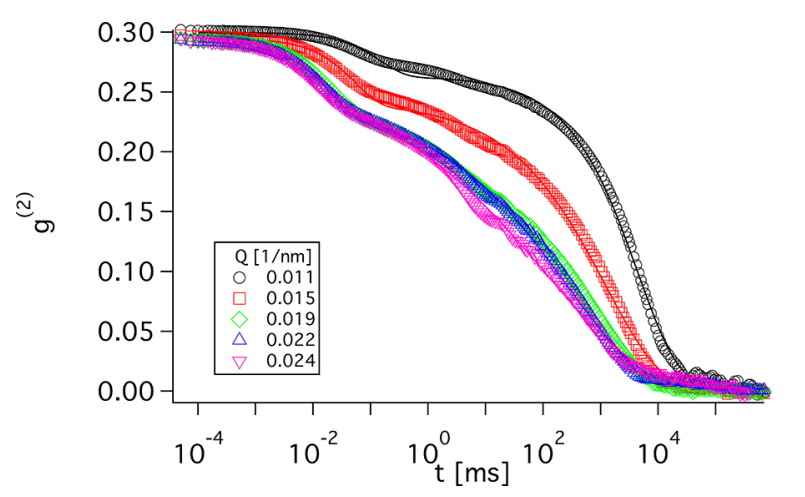

Figure 4. Intensity autocorrelation functions of $1 \mathrm{wt} \% \mathrm{JR} 400,1.5$ $\mathrm{mM} \operatorname{SDS}(Z=7)$ at $Q$ indicated in the graph; all curves show two relaxation modes.

observed previously for such systems, ${ }^{36,37}$ and can be described in the framework of the mode coupling theory, ${ }^{67}$ where the relaxation time of the slow mode $\tau_{2}$ should scale as $\tau_{2} \sim \tau_{1}{ }^{1 / \alpha}$. Assuming diffusive behavior for the fast mode $\tau_{1}\left(\tau_{1}=\frac{1}{D_{1} Q^{2}}\right)$ and converting $\tau_{2}$ to an apparent diffusion coefficient, $D_{2}$ should scale as $D_{2} \sim Q^{1 / \alpha}$.

The diffusion coefficients obtained for the fast mode $D_{1}$ is almost constant, increasing only slightly with $Q$ and their order of magnitude is in reasonably good agreement with the diffusion coefficient of the $48 \mathrm{~nm}$ long rodlike particles (see Figure S6), which would be about $2 \AA^{2} /$ ns. The higher values at lower concentrations (high $Z$ ) can be explained by the fact that the scattering does not exclusively stem from the rodlike particles, but the free PE contributes as well with a faster relaxation.

The slow mode, which should stem from the large clusters like the mode observed in FCS, shows a quantitatively different behavior compared to the FCS measurements (see Figure 5). The diffusion coefficient of the slow mode is on the order of 1 $\times 10^{-3}$ to $1 \times 10^{-5} \AA^{2} / \mathrm{ns}$, which is 1 to 3 orders of magnitude below the values found in FCS. In addition, the values 


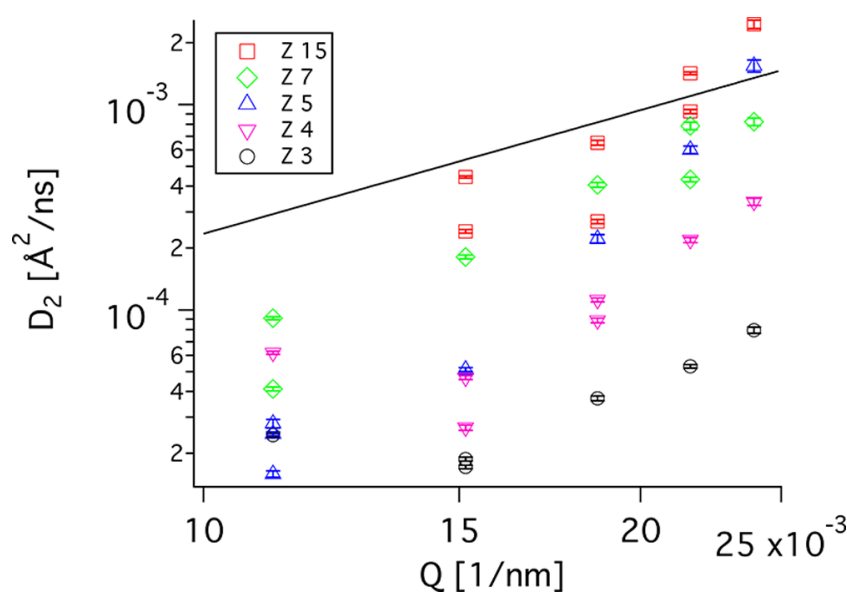

Figure 5. Diffusion coefficients of the slow mode observed in DLS $\mathrm{D}_{2}$ for samples with $1 \mathrm{wt} \%$ JR 400 and different amounts of SDS noted in the graph; the values are significantly slower than those observed in FCS. The straight line indicates scaling with $Q^{2}$, which was chosen as an intermediate value between 1.7 and 3.3 .

continuously decrease as the surfactant concentration is increased ( $Z$ is decreased). As opposed to the FCS correlation function, the slow mode in DLS shows a wide distribution of relaxation rates with a stretch parameter $\alpha$ between 0.3 and 0.6 (Figure S7), which slightly increases with decreasing $Q$ so that the decreasing trend in $D_{2}$ with decreasing $Q$ is somewhat compensated in the averaged value $D_{2 A v}$ (see Figure S8). Following the mode coupling theory, these values of $\alpha$ should result in a relatively strong scaling of $D_{2}$ with $Q$ with exponents between 1.7 and 3.3 which seems to be the case (see Figure 5). The amplitude of the fast mode is found to be relatively small and constant with $Q$ and has values on the order $0.05-0.1$ (see Figure S9). The values observed for $D_{2 \mathrm{Av}}$ from $1 \times 10^{-4}$ to $<1$ $\times 10^{-5} \AA^{2} / \mathrm{ns}$ would correspond to hydrodynamic radii of tens to thousands of micrometers which would already correspond to lengths on the order of the sample size. This clearly contradicts the findings from FCS with structures on the order of some $100 \mathrm{~nm}$. Accordingly, both methods, DLS and FCS, yield largely different size estimates for the relevant structures here, which are not to be reconciled within a simple structural picture.

USANS. To resolve this discrepancy, we performed USANS measurements as $300 \mathrm{~nm}$ would be a length scale easily observed with USANS, while tens of micrometers would even exceed the size range of this method.

Combined USANS and SANS curves of SDS/JR 400 with $Z$ $=3$ for both deuterated and hydrogenated SDS can be seen in Figure 6. At $Q>0.11 / \mathrm{nm}$ the previously observed rodlike structures are reproduced, and at $Q<0.011 / \mathrm{nm}$ a $Q^{-4}$ slope is observed for both deuterated and hydrogenated SDS, where the intensity is significantly higher for the hydrogenated SDS, which is in agreement with the findings from DLS, indicating very large polydisperse structures which are composed of both the PE and the surfactant. Therefore, the possibility that the surfactant does not take part in the very large structures, and the FCS mode is faster as the hydrophobic dye is exclusively located in the surfactant aggregates can be ruled out.

Knowing the scattering length densities of the components (surfactant, PE, and, judging from the transparent appearance of the samples, a considerable amount of water) and assuming that the structures formed with deuterated SDS and hydro-

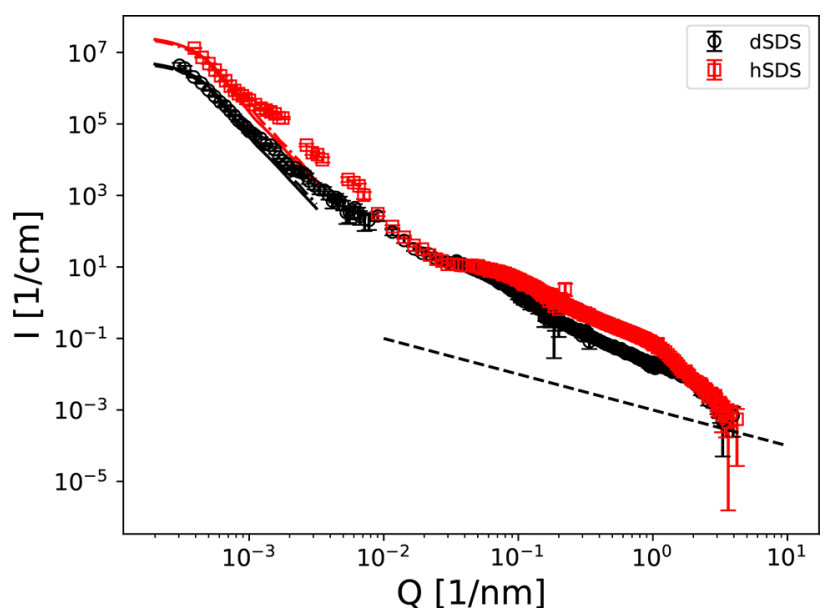

Figure 6. Combined SANS and USANS curves for 1 wt $\%$ JR 400 PESCs with $3.3 \mathrm{mM}$ SDS (chareg ratio $Z=3$ ) with both deuterated (black) and hydrogenated (red) SDS; straight lines: $4000 \mathrm{~nm}$ spheres with 30\% relative polydispersity; dash-dotted lines: $600 \mathrm{~nm}$ spheres with $350 \%$ relative standard deviation (see eq 1 , low $Q$ ), $Q^{-1}$ (high $Q)$. No plateau is reached at low $Q$.

genated SDS are identical, the ratio of the intensities depends exclusively on the ratio $r$ of the square of the scattering length density differences between the clusters and the solvent:

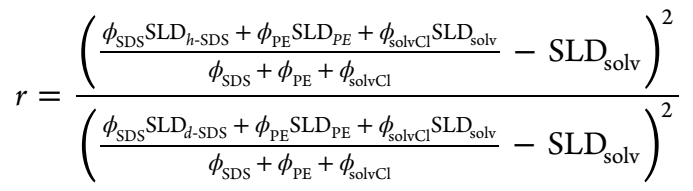

where $\phi$ are the volume fractions, SLD are the scattering length densities, and the indexes $\mathrm{PE}$, solv, solvCl, and $(h / d)$ SDS represent the polyelectrolyte, the solvent, the solvent in the clusters, and (hydrogenated/deuterated) SDS, where we assume that the volume fraction is identical for both. As the smaller rodlike aggregates are the building blocks for the clusters and the critical aggregation concentration for ionic surfactants with oppositely charged polyelectrolyte are extremely low, ${ }^{26,64,68}$ it is safe to assume that almost all of the surfactant is involved in the formation of the clusters. The dependence on $\phi_{\text {solvCl }}$ cancels out, and the ratio is determined by the amount of JR 400 in the clusters:

$$
\phi_{\mathrm{PE}}=\frac{\phi_{\mathrm{SDS}}\left(\sqrt{r} \Delta \mathrm{SLD}_{d-\mathrm{SDS}}-\Delta \mathrm{SLD}_{h-\mathrm{SDS}}\right)}{\Delta \mathrm{SLD}_{\mathrm{PE}}(1-\sqrt{r})}
$$

where $\Delta \mathrm{SLD}_{x}$ is the difference in scattering length density between component $x$ and solvent. Experimentally, we observe an intensity ratio between the sample with $h$-SDS and $d$-SDS of about $r=5$, and using eq 22 with the appropriate values we obtain $\phi_{\mathrm{PE}}=\frac{0.001\left(\sqrt{5} \cdot 3.75 \times 10^{-5}-6 \times 10^{-4}\right)}{2.65 \times 10^{-4}(1-\sqrt{5})}=0.0016$, which is significantly less than the macroscopic volume fraction $\phi_{\mathrm{PE}}$ of 0.0065 and is close to charge equilibrium and the composition in the rodlike aggregates. ${ }^{44}$ With a density of JR $400 \rho_{\mathrm{JR} 400}=1.66$ and a molecular weight per charge of $M_{\mathrm{JR} 400}=$ $1000 \mathrm{~g} / \mathrm{mol}$, the concentration of JR 400 charges in the clusters is $\mathcal{c}_{\mathrm{PE}}=0.0016 \cdot 1.66 \mathrm{~g} / \mathrm{mL} / 1000 \mathrm{~g} / \mathrm{mol}=2.7 \mathrm{mmol} / \mathrm{L}$, which is only a little less than the $3.3 \mathrm{mmol} / \mathrm{L}$ of SDS in the sample, and the resulting charge ratio in the clusters is 0.82 ; i.e., they are largely neutralized. 
Having only the limiting $Q^{-4}$ part of the clusters, it is impossible to determine a precise length scale for the clusters. However, we can take a look at some limiting cases, assuming for simplicity and lacking any better indication a spherical geometry of the clusters. It is possible to estimate a minimum size of the clusters, assuming the onset of the form factor plateau starts at $Q$ right below the accessible range. Additionally, this yields a minimum polydispersity required to make the form factor oscillations vanish at this size. The full lines at low $Q$ in Figure 6 were generated using eqs 1 to 3 and 5 assuming an average size of $4000 \mathrm{~nm}$ and a relative standard deviation of $30 \%$. To get the correct intensity, it had to be assumed that in addition to the volume fractions of SDS $\left(\phi_{\mathrm{SDS}}\right.$ $=0.001)$ and $\mathrm{JR} 400\left(\phi_{\mathrm{PE}}=0.0016\right.$, following eq 22) an additional volume fraction of water $\phi_{\text {solvCl }}=0.2$ had to be integrated in the clusters, meaning that they consist of almost 99\% water. The corresponding Porod constants $B_{\mathrm{s}}$ are $0.5 \times$ $10^{-14}$ and $2.5 \times 10^{-14} 1 / \mathrm{nm}^{5}$ for the deuterated and the hydrogenated sample. Assuming no water in the clusters following eq 7 , this corresponds to a size of $\left\langle R^{3}\right\rangle /\left\langle R^{2}\right\rangle=$ $6 \pi \phi \Delta \mathrm{SLD}^{2} / B_{\mathrm{s}}=6 \pi \cdot 0.0026 \cdot\left(3.94 \times 10^{-4} 1 / \mathrm{nm}^{2}\right)^{2} / 2.5 \times 10^{-14}$ $1 / \mathrm{nm}^{5}=304 \mu \mathrm{m}$ as an upper limit and $3.9 \mu \mathrm{m}$ as a lower limit if we assume a water content of $99 \%$, resulting in $\Delta$ SLD $=0.05$ $\times 10^{-4} 1 / \mathrm{nm}^{2}$. For monodisperse clusters, these values would immediately correspond to the cluster size.

Another estimate for the polydispersity of the clusters comes from the stretch parameter of the slow mode in DLS. Following eq 14, the relative standard deviation for a given $\alpha$ is $\sqrt{\Gamma\left(\frac{2}{\alpha}\right)-\left(\Gamma\left(\frac{1}{\alpha}\right)\right)^{2}} / \Gamma\left(\frac{1}{\alpha}\right)$. With a value of $\alpha=0.4$ as found here, this corresponds to an extremely high relative standard deviation of 3.5. This value is certainly overestimating the real value as the DLS signal involves higher moments of the distribution. The problem of converting polydispersity between DLS and SANS has been dealt with in detail before ${ }^{69,70}$ However, the case is a little more complicated here, as we are neither in the low- $Q$ nor in the high- $Q$ limit if we assume that we are dealing with a rather broad distribution of cluster sizes. Therefore, we simply use the value from DLS as an upper limit. Starting from eqs 4 and 7 , we get $R_{\mathrm{m}}=\left\langle R^{3}\right\rangle /\left\langle R^{2}\right\rangle \exp \left(-\frac{5}{2} \sigma^{2}\right)$ and $\sigma=\sqrt{\log \left(\mathrm{sd}^{2}+1\right)}$, with the relative standard deviation sd, resulting in a mean cluster size $\left\langle R^{1}\right\rangle=\left\langle R^{3}\right\rangle /\left\langle R^{2}\right\rangle \exp \left(-2 \log \left(\mathrm{sd}^{2}+1\right)\right)$. By use of the previously obtained values for $\left\langle R^{3}\right\rangle /\left\langle R^{2}\right\rangle$ of 304 and $3.9 \mu \mathrm{m}$ and relative standard deviations of 0.3 and 3.5, the resulting mean cluster sizes are 256 and $1.7 \mu \mathrm{m}$ in the dry case and 3.3 $\mu \mathrm{m}$ and $22 \mathrm{~nm}$ assuming $99 \% \mathrm{D}_{2} \mathrm{O}$ in the clusters, while the last value is clearly unrealistic and fails to reproduce the low- $Q$ behavior in Figure 6. The dash-dotted curves at low $Q$ in Figure 6 have been generated in the same fashion as the full lines, except that a mean size of $600 \mathrm{~nm}$ has been used instead of $4 \mu \mathrm{m}$ with a relative polydispersity of 3.5. Taking 3.5 as the maximum polydispersity $600 \mathrm{~nm}$ is the lower limit for the mean size of the clusters, as otherwise a plateau would become visible at low $Q$. Given the extremely large polydispersity, this would still mean that a large fraction of the clusters would be micrometer sized. Realistically, this overestimates the polydispersity and underestimates the average size.

\section{DISCUSSION}

We measured the DLS and FCS correlation functions of highly viscous polyelectrolyte-surfactant complexes consisting of the cellulose-based polycation JR 400 and the anionic surfactant SDS. It is known from NSE and SANS measurements that they aggregate into mixed rodlike aggregates with a length of $\sim 50$ $\mathrm{nm}$ and a radius of $<2 \mathrm{~nm}$, which interconnect multiple PE chains, thereby causing the increase in viscosity.

DLS showed a fast relaxation mode corresponding to the diffusion of those rodlike particles, but another much slower mode was observed as well. FCS measurements yielded a mode significantly slower than what would be expected for $50 \mathrm{~nm}$ rodlike particles but significantly faster than the slow mode observed in DLS.

Our dynamic measurements were complemented by USANS measurements to shed light on the structure on long length scales, and it was found that extremely large clusters are formed which exceed even the size accessible to USANS. By combining measurements with hydrogenated and deuterated SDS, we could conclude that only a relatively small fraction of polyelectrolyte is incorporated in the clusters, and their composition is relatively close to charge equilibrium. While it is not possible to determine a precise length scale of the clusters from the measurements, we can estimate that their size must be between a few micrometers and a few hundred micrometers. The larger value would mean that there is no additional water in the clusters, which seems unlikely judging from the transparent appearance of the samples, and the true size is most likely closer to the lower estimate.

This still leaves the question of the large discrepancy between the diffusion coefficients from DLS and FCS open, and a possible explanation might be the different weighting in both methods. The different weighting of contributions in DLS and FCS can have a significant influence on the observed average diffusion coefficient. While FCS measures a numberaverage, contributions in DLS are weighted with their scattering power, which corresponds to a $z$-average diffusion coefficient, if $R<1 / Q$. For polydisperse samples, this can lead to significantly larger values than the number-average. However, the condition $R<1 / Q$ is not fulfilled here, and at high $Q$ the contribution of a particle is proportional to its surface to volume ratio $\left\langle R^{(2)}\right\rangle /\left\langle R^{(3)}\right\rangle$, which is less for larger particles. We performed some simple simulations (see the Supporting Information, section III) which show that the combination of different weighting and polydispersity can lead to a significant difference between the average values observed by DLS and FCS but not 3 orders of magnitude and more. Therefore, the different weighting of contributions cannot explain the discrepancies between the results from DLS and FCS.

Another factor that can lead to large differences in the observed diffusion coefficient is the time scale of the experiment. ${ }^{71}$ If the diffusing objects can change between different states with different diffusion coefficients as for example being part of a cluster or not or moving in a heterogeneous environment where their diffusion coefficient changes as a function of their location, the time scale on which these changes happen starts playing a role. In the limit of short experimental times and long switching times between states, the measured diffusion coefficient behaves as if no switching took place:

$$
C(t)=\int_{0}^{\infty} f(\tau) \exp (-t / \tau) \mathrm{d} \tau
$$

where $C$ is a correlation function and $f$ is a distribution over the relaxation times, which are proportional to the radius for 
translational diffusion (see eq 16). In the limit of long experimental time scales compared to the switching time, the diffusion coefficient is averaged as the observed particles spent a certain amount of time in either state during the observation:

$$
C(t)=\exp \left(-\int_{0}^{\infty} f(\tau) t / \tau\right) \mathrm{d} \tau
$$

While the time scales of the measurement in DLS and FCS are similar, DLS measures the coherent scattering signal, while FCS observes the self-correlation of individual particles, which would correspond to the incoherent signal in neutron scattering. So not only is the scattering of larger particles emphasized in DLS, but also the relevant time scale is different. While in DLS the relevant time scale is the lifetime of a cluster, it is the residence time of an individual aggregate in a cluster (or even the lifetime of an aggregate itself or the residence time of the dye in the aggregate) for FCS, and the former can be significantly longer than the latter. This would also explain why only a single mode was observed in FCS, while the decay in DLS is bimodal as eq 24 remains monomodal even for multiple different diffusion coefficients. On the basis of this assumption, we can also conclude that the residence time of an individual aggegate in a cluster is shorter than the roughly $10 \mathrm{~ms}$ observed as relaxation time in FCS, and the lifetime of a cluster must be at least on the order of some seconds, according to the DLS data.

Assuming that the rodlike aggregates in which the hydrophobic dye resides either are or are not part of a cluster and that the switching time between the states is short, the measured diffusion coefficient is an average between the diffusion coefficient of the rodlike aggregates known from our previous NSE experiments of about $2 \AA^{2} / \mathrm{ns}$ and the much slower value from the clusters $D_{\mathrm{Av}}=x D_{\text {cluster }}+(1-x) D_{\text {rod }}$. This allows us to calculate the fraction of time spent in clusters and equivalently the fraction of aggregates which are found in clusters from the average diffusion coefficient measured with FCS: $x=\frac{D_{\mathrm{Av}}-D_{\text {rod }}}{D_{\text {cluster }}-D_{\text {rod }}}$. In the limiting case of using $D_{\text {cluster }}=$ $0 \AA^{2} / \mathrm{ns}$, this leads to $x=\frac{0.04-2}{0-2}=0.98$ as the lower estimate for the fraction of rodlike aggregates in the clusters. This also justifies our assumption in the analysis of the USANS data that all the surfactant is found in the clusters, and we have satisfactorily explained the differences in the relaxation behavior between FCS and DLS. We have neglected any contribution from free dye, which would add another fast contribution to $D_{\mathrm{Av}}$, resulting in an even higher value of $x$.

\section{SUMMARY}

In summary, we found large, micrometer sized clusters in viscous PESCs formed from SDS and JR 400 which consist of the rodlike nanometers sized mixed aggregates we found previously. ${ }^{43,44}$ The clusters have a very high water content on the order of $99 \%$, so they should be thought of as regions of higher concentration rather than dense objects as depicted in Figure 7. This is similar to findings reported for mixtures of hyaluronic acid and $\mathrm{TTAB}^{28}$ with the difference that the clusters observed there had sizes on the order of a few $100 \mathrm{~nm}$. The fact that the JR 400/SDS clusters are at least an order of magnitude larger might be related to their high viscosity. Furthermore, it was found that almost all of the nanometer sized aggregates are part of the clusters, and therefore almost all of the surfactant is located in them. For a charge ratio of $Z=$

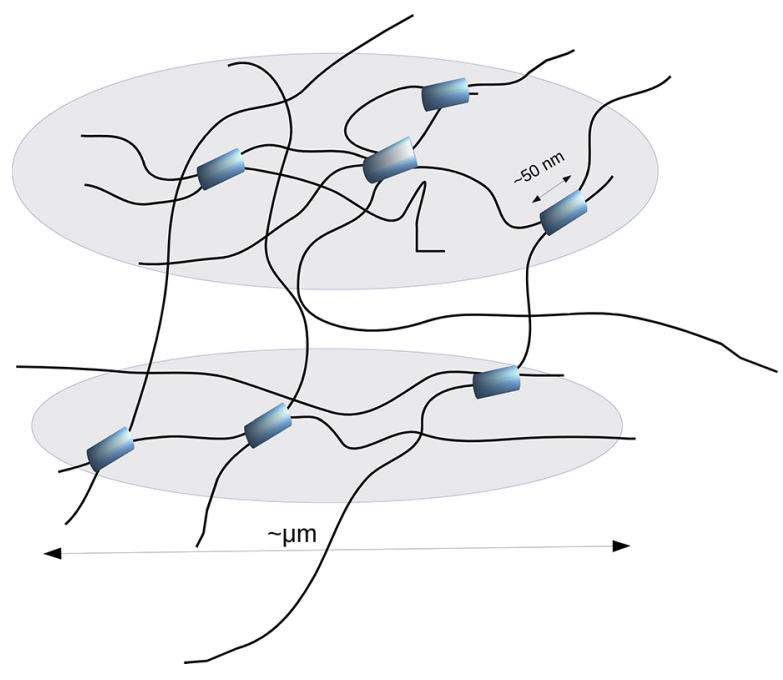

Figure 7. Drawing of the clusters (gray background) which are regions with a somewhat higher density of PE (black lines) and contain almost all of the surfactant aggregates (blue rods) but nevertheless consist mostly of water.

3, where USANS measurements were performed, it was found that about a quarter of the JR 400 is part of the clusters, which is relatively close to charge equilibrium between surfactant and PE. It is reasonable to assume that this ratio is maintained for lower surfactant concentrations.

The slow diffusion observed in FCS and DLS is attributed to movements of these clusters. The large quantitative difference between the results from FCS and DLS is explained in terms of the difference between short and long time behavior of the diffusion. While the correlation times are similar in FCS and DLS, the relevant time scales are different. While in FCS the relevant time scale is the residence time of a single aggregate in a cluster, it is the lifetime of the whole cluster in DLS. Judging by our results, the former is short compared to the diffusion times in FCS while the latter is long compared to the time scale in DLS.

\section{ASSOCIATED CONTENT}

\section{Supporting Information}

The Supporting Information is available free of charge on the ACS Publications website at DOI: 10.1021/acs.macromol.8b02611.

Details on the calculation of the length of the aggregates from NSE data, additional figures and simulations (PDF)

\section{AUTHOR INFORMATION}

\section{Corresponding Authors}

*E-mail: hoffmann@ill.fr.

*E-mail: michael.gradzielski@tu-berlin.de.

ORCID

Ingo Hoffmann: 0000-0001-7178-6467

Michael Gradzielski: 0000-0002-7262-7115

Notes

The authors declare no competing financial interest.

\section{ACKNOWLEDGMENTS}

Financial support from the BMBF project 05K13KT1 is gratefully acknowledged as well as allocation of beamtime by 
ILL NCNR. Access to USANS BT5 and NGB30SANS was provided by the Center for High Resolution Neutron Scattering, a partnership between the National Institute of Standards and Technology and the National Science Foundation under Agreement DMR-1508249. Certain commercial equipment, instruments, or materials are identified in this paper to foster understanding. Such identification does not imply recommendation or endorsement by the National Institute of Standards and Technology, nor does it imply that the materials or equipment identified are necessarily the best available for the purpose. Raw data of the NSE measurements is available under dx.doi.org/10.5291/ILLDATA.TEST-2150.

\section{REFERENCES}

(1) Schaffer, J. F.; Woodhams, R. T. Polyelectrolyte Builders as Detergent Phosphate Replacements. Ind. Eng. Chem. Prod. Res. Dev. 1977, 16, 3-11.

(2) Taugbøl, K.; Ly, T. V.; Austad, T. Chemical flooding of oil reservoirs 3. Dissociative surfactant-polymer interaction with a positive effect on oil recovery. Colloids Surf., A 1995, 103, 83-90.

(3) Guzmán, E.; Llamas, S.; Maestro, A.; Fernández-Peña, L.; Akanno, A.; Miller, R.; Ortega, F.; Rubio, R. G. Polymer-surfactant systems in bulk and at fluid interfaces. Adv. Colloid Interface Sci. 2016, 233, 38-64.

(4) Goddard, E. D.; Hannan, R. B. Cationic Polymer/Anionic Surfactant Interactions. J. Colloid Interface Sci. 1976, 55, 73-79.

(5) Piculell, L.; Lindman, B. Association and Segregation in Aqueous Polymer/Polymer, Polymer Surfactant, and Surfactant Surfactant Mixtures - Similarities and Differences. Adv. Colloid Interface Sci. 1992, 41, 149-178.

(6) Kästner, U.; Hoffmann, H.; Donges, R.; Ehrler, R. Interactions between modified hydroxyethyl cellulose (HEC) and surfactants. Colloids Surf., A 1996, 112, 209-225.

(7) Kwak, J. C. T., Ed.; Polymer-Surfactant Systems; Marcel Dekker, Inc.: 1998; Vol. 77.

(8) Kosmella, S.; Kötz, J.; Shirahama, K.; Liu, J. Cooperative Nature of Complex Formation in Mixed Polyelectrolyte-Surfactant Systems. J. Phys. Chem. B 1998, 102, 6459-6464.

(9) Marques, E. F.; Regev, O.; Khan, A.; Miguel, M. d. G.; Lindman, B. Interactions between Catanionic Vesicles and Oppositely Charged Polyelectrolytes Phase Behavior and Phase Structures. Macromolecules 1999, 32, 6626-6637.

(10) Yamaguchi, Y.; Inaba, Y.; Uchiyama, H.; Kunieda, H. Anomalous phase behavior of water-soluble polyelectrolyte and oppositely charged surfactants. Colloid Polym. Sci. 1999, 277, 11171124.

(11) Svensson, A.; Sjöström, J.; Scheel, T.; Piculell, L. Phases and structures of a polyion-surfactant ion complex salt in aqueous mixtures: cationic hydroxyethyl cellulose with dodecylsulfate counterions. Colloids Surf., A 2003, 228, 91-106.

(12) Trabelsi, S.; Guillot, S.; Raspaud, E.; Delsanti, M.; Langevin, D.; Boué, F. New nano- and microparticles with a liquid-crystal-like interior. Adv. Mater. 2006, 18, 2403-2406.

(13) Bain, C. D.; Claesson, P. M.; Langevin, D.; Meszaros, R.; Nylander, T.; Stuben-rauch, C.; Titmuss, S.; Von Klitzing, R. Complexes of surfactants with oppositely charged polymers at surfaces and in bulk. Adv. Colloid Interface Sci. 2010, 155, 32-49.

(14) Dos Santos, S.; Gustavsson, C.; Gudmundsson, C.; Linse, P.; Piculell, L. When Do Water-Insoluble Polyion - Surfactant Ion Complex Salts "Redissolve" by Added Excess Surfactant? Langmuir 2011, 27, 592-603.

(15) Lam, V. D.; Walker, L. M. A pH-Induced Transition of Surfactant-Polyelectrolyte Aggregates from Cylindrical to String-ofPearls Structure. Langmuir 2010, 26, 10489-10496.

(16) Penott-Chang, E. K.; Ruppel, M.; Pergushov, D. V.; Zezin, A. B.; Müller, A. H. Interpolyelectrolyte complexes of diblock copolymers via interaction of complementary polyelectrolytesurfactant complexes in chloroform. Polymer 2011, 52, 4296-4302.

(17) Moroz, E.; Zakharova, J.; Sergeyev, V.; Zezin, A. Ternary complexes polyelectrolyte- oppositely charged surfactant-carbon nanotubes: Formation and properties. Dokl. Phys. Chem. 2011, 441, 212-214.

(18) Mohr, A.; Nylander, T.; Piculell, L.; Lindman, B.; Boyko, V.; Bartels, F. W.; Liu, Y.; Kurkal-Siebert, V. Mixtures of Cationic Copolymers and Oppositely Charged Surfactants: Effect of Polymer Charge Density and Ionic Strength on the Adsorption Behavior at the Silica-Aqueous Interfaces. ACS Appl. Mater. Interfaces 2012, 4, 15001511.

(19) Štěpánek, M.; Škvarla, J.; Uchman, M.; Procházka, K.; Angelov, B.; Kováčik, L.; Garamus, V. M.; Mantzaridis, C.; Pispas, S. Wormlike core-shell nanoparticles formed by co-assembly of double hydrophilic block polyelectrolyte with oppositely charged fluorosurfactant. Soft Matter 2012, 8, 9412-9417.

(20) Campbell, R. A.; Yanez Arteta, M.; Angus-Smyth, A.; Nylander, T.; Noskov, B. A.; Varga, I. Direct Impact of Nonequilibrium Aggregates on the Structure and Morphology of Pdadmac/SDS Layers at the Air/Water Interface. Langmuir 2014, 30, 8664-8674.

(21) Fegyver, E.; Meszaros, R. The impact of nonionic surfactant additives on the nonequilibrium association between oppositely charged polyelectrolytes and ionic surfactants. Soft Matter 2014, 10, $1953-1962$.

(22) Weston, J. S.; Harwell, J. H.; Shiau, B. J.; Kabir, M. Disrupting admicelle formation and preventing surfactant adsorption on metal oxide surfaces using sacrificial polyelectrolytes. Langmuir 2014, 30, 6384-6388.

(23) Chiappisi, L.; Simon, M.; Gradzielski, M. Toward Bioderived Intelligent Nanocarriers for Controlled Pollutant Recovery and $\mathrm{pH}$ Sensitive Binding. ACS Appl. Mater. Interfaces 2015, 7, 6139-6145.

(24) Kötz, J.; Kosmella, S.; Beitz, T. Self-assembled polyelectrolyte systems. Prog. Polym. Sci. 2001, 26, 1199-1232.

(25) Berret, J. Controlling electrostatic co-assembly using ioncontaining copolymers: From surfactants to nanoparticles. Adv. Colloid Interface Sci. 2011, 167, 38-48.

(26) Chiappisi, L.; Hoffmann, I.; Gradzielski, M. Complexes of oppositely charged polyelectrolytes and surfactants - recent developments in the field of biologically derived polyelectrolytes. Soft Matter 2013, 9, 3896-3909.

(27) Chronakis, I. S.; Alexandridis, P. Rheological Properties of Oppositely Charged Polyelectrolyte-Surfactant Mixtures: Effect of Polymer Molecular Weight and Surfactant Architecture. Macromolecules 2001, 34, 5005-5018.

(28) Buchold, P.; Schweins, R.; Di, Z.; Gradzielski, M. Structural behaviour of sodium hyaluronate in concentrated oppositely charged surfactant solutions. Soft Matter 2017, 13, 2253-2263.

(29) Plucktaveesak, N.; Konop, A. J.; Colby, R. H. Viscosity of Polyelectrolyte Solutions with Oppositely Charged Surfactant. J. Phys. Chem. B 2003, 107, 8166-8171.

(30) Ikkala, O.; Ten Brinke, G. Hierarchical self-assembly in polymeric complexes: Towards functional materials. Chem. Commun. 2004, 2131-2137.

(31) Berret, J. Evidence of overcharging in the complexation between oppositely charged polymers and surfactants. J. Chem. Phys. 2005, 123, 164703.

(32) Trabelsi, S.; Langevin, D. Co-adsorption of CarboxymethylCellulose and Cationic Surfactants at the Air-Water Interfaces. Langmuir 2007, 23, 1248-1252.

(33) Trabelsi, S.; Guillot, S.; Ritacco, H.; Boué, F.; Langevin, D. Nanostructures of colloidal complexes formed in oppositely charged polyelectrolyte/surfactant dilute aqueous solutions. Eur. Phys. J. E: Soft Matter Biol. Phys. 2007, 23, 305-311.

(34) Hajduová, J.; Procházka, K.; Šlouf, M.; Angelov, B.; Mountrichas, G.; Pispas, S.; Štepánek, M. Polyelectrolyte-Surfactant Complexes of Poly[3,5-bis(dimethylaminomethyl)-4-hydroxystyrene]-block-poly(ethylene oxide) and Sodium Dodecyl Sulfate: Anomalous Self-Assembly Behavior. Langmuir 2013, 29, 5443-5449. 
(35) Chiappisi, L.; Prévost, S.; Grillo, I.; Gradzielski, M. From Crab Shells to Smart Systems: Chitosan-Alkylethoxy Carboxylate Complexes. Langmuir 2014, 30, 10608-10616.

(36) Tsianou, M.; Kjøniksen, A. L.; Thuresson, K.; Nyström, B. Light Scattering and Viscoelasticity in Aqueous Mixtures of Oppositely Charged and Hydrophobically Modified Polyelectrolytes. Macromolecules 1999, 32, 2974-2982.

(37) Hoffmann, I.; Heunemann, P.; Prévost, S.; Schweins, R.; Wagner, N. J.; Gradzielski, M. Self-Aggregation of Mixtures of Oppositely Charged Polyelectrolytes and Surfactants Studied by Rheology, Dynamic Light Scattering and Small-Angle Neutron Scattering. Langmuir 2011, 27, 4386-4396.

(38) Hoffmann, I.; Prévost, S.; Medebach, M.; Rogers, S. E.; Wagner, N. J.; Gradzielski, M. Control of Rheological Behaviour with Oppositely Charged Polyelectrolyte Surfactant Mixtures. Tenside, Surfactants, Deterg. 2011, 48, 488-494.

(39) Uchman, M.; Hajduová, J.; Vlassi, E.; Pispas, S.; Appavou, M.; Štěpánek, M. Self- and co-assembly of amphiphilic gradient polyelectrolyte in aqueous solution: Interaction with oppositely charged ionic surfactant. Eur. Polym. J. 2015, 73, 212-221.

(40) Drifford, M.; Dalbiez, J. Dynamics of polyelectrolyte solutions by light scattering. J. Phys., Lett. 1985, 46, 311-319.

(41) Nierling, W.; Nordmeier, E. Studies on Polyelectrolyte Solutions VII. Fast, Heterogeneous, and Slow Diffusion Modes of Poly(diallyl-N,N-dimethylammonium chloride) in Aqueous Alcoholic Salt Solvents. Polym. J. 1997, 29, 795-806.

(42) Ermi, B. D.; Amis, E. J. Domain Structures in Low Ionic Strength Polyelectrolyte Solutions. Macromolecules 1998, 31, 73787384.

(43) Hoffmann, I.; Farago, B.; Schweins, R.; Falus, P.; Sharp, M.; Gradzielski, M. Structure and dynamics of polyelectrolytes in viscous polyelectrolyte-surfactant complexes at the mesoscale. Europhys. Lett. 2013, 104, 28001.

(44) Hoffmann, I.; Farago, B.; Schweins, R.; Falus, P.; Sharp, M.; Prévost, S.; Gradzielski, M. On the mesoscopic origins of high viscosities in some polyelectrolyte-surfactant mixtures. J. Chem. Phys. 2015, 143, No. 074902.

(45) Broersma, S. Rotational Diffusion Constant of A Cylindrical Particle. J. Chem. Phys. 1960, 32, 1626-1631.

(46) Kline, S. R. Reduction and analysis of SANS and USANS data using IGOR Pro. J. Appl. Crystallogr. 2006, 39, 895-900.

(47) Barker, J. G.; Glinka, C. J.; Moyer, J. J.; Kim, M. H.; Drews, A. R.; Agamalian, M. Design and performance of a thermal-neutron double-crystal diffractometer for USANS at NIST. J. Appl. Crystallogr. 2005, 38, 1004-1011.

(48) Glatter, O., Kratky, O., Eds.; Small Angle X-ray Scattering; Academic Press Inc.: 1982.

(49) Beaucage, G.; Kammler, H. K.; Pratsinis, S. E. Particle size distributions from small- angle scattering using global scattering functions. J. Appl. Crystallogr. 2004, 37, 523-535.

(50) Mezei, F. In Lecture Notes in Physics; Mezei, F., Ed.; Springer: Heidelberg, 1980; Vol. 128, pp 1-26.

(51) Schleger, P.; Alefeld, B.; Barthelemy, J.; Ehlers, G.; Farago, B.; Giraud, P.; Hayes, C.; Kollmar, A.; Lartigue, C.; Mezei, F.; Richter, D. The long-wavelength neutron spin-echo spectrometer IN15 at the Institut Laue-Langevin. Phys. B 1997, 241-243, 164-165.

(52) Richter, D.; Monkenbusch, M.; Arbe, A.; Colmenero, J. Neutron spin echo in polymer systems. Adv. Polym. Sci. 2005, 174, 1221.

(53) Holderer, O.; Monkenbusch, M.; Schätzler, R.; Kleines, H.; Westerhausen, W.; Richter, D. The JCNS neutron spin-echo spectrometer J-NSE at the FRM II. Meas. Sci. Technol. 2008, 19, No. 034022.

(54) Farago, B. Recent developments and applications of NSE in soft matter. Curr. Opin. Colloid Interface Sci. 2009, 14, 391-395.

(55) Colmenero, J.; Arbe, A. Recent progress on polymer dynamics by neutron scattering: From simple polymers to complex materials. $J$. Polym. Sci., Part B: Polym. Phys. 2013, 51, 87-113.
(56) Hoffmann, I. Neutrons for the study of dynamics in soft matter systems. Colloid Polym. Sci. 2014, 292, 2053-2069.

(57) Farago, B.; Falus, P.; Hoffmann, I.; Gradzielski, M.; Thomas, F.; Gomez, C. The IN15 upgrade. Neutron News 2015, 26, 15-17.

(58) Berne, B. J.; Pecora, R. Dynamic Light Scattering With Applications to Chemistry, Biology and Physics, reprint ed.; Dover Publications: 2000

(59) De Gennes, P. G. Liquid dynamics and inelastic scattering of neutrons. Physica 1959, 25, 825-839.

(60) Petrášek, Z.; Schwille, P. Precise Measurement of Diffusion Coefficients using Scanning Fluorescence Correlation Spectroscopy. Biophys. J. 2008, 94, 1437-1448.

(61) Malo De Molina, P.; Appavou, M.; Gradzielski, M. Oil-in-water microemulsion droplets of TDMAO/decane interconnected by the telechelic C18-EO150-C18: clustering and network formation. Soft Matter 2014, 10, 5072-5084.

(62) Banks, D. S.; Fradin, C. Anomalous Diffusion of Proteins Due to Molecular Crowding. Biophys. J. 2005, 89, 2960-2971.

(63) Weiss, M.; Elsner, M.; Kartberg, F.; Nilsson, T. Anomalous Subdiffusion Is a Measure for Cytoplasmic Crowding in Living Cells. Biophys. J. 2004, 87, 3518-3524.

(64) Li, D.; Kelkar, M. S.; Wagner, N. J. Phase Behavior and Molecular Thermodynamics of Coacervation in Oppositely Charged Polyelectrolyte/Surfactant Systems: A CationicPolymer JR 400 and Anionic Surfactant SDS Mixture. Langmuir 2012, 28, 10348-10362.

(65) Thuresson, K.; Nilsson, S.; Lindman, B. Effect of Hydrophobic Modification on Phase Behavior and Rheology in Mixtures of Oppositely Charged Polyelectrolytes. Langmuir 1996, 12, 530-537.

(66) Hoffmann, I.; Simon, M.; Farago, B.; Schweins, R.; Falus, P.; Holderer, O.; Gradzielski, M. Structure and dynamics of polyelectrolyte surfactant mixtures under conditions of surfactant excess. $J$. Chem. Phys. 2016, 145, 124901.

(67) Ngai, K. L.; Rajagopal, A. K.; Teitler, S. Slowing Down Of Relaxation In A Complex System By Constraint Dynamics. J. Chem. Phys. 1988, 88, 5086-5094.

(68) Jain, N.; Trabelsi, S.; Guillot, S.; McLoughlin, D.; Langevin, D.; Letellier, P.; Turmine, M. Critical Aggregation Concentration in Mixed Solutions of Anionic Polyelectrolytes and Cationic Surfactants. Langmuir 2004, 20, 8496-8503.

(69) Thomas, J. C. The determination of log normal particle size distributions by dynamic light scattering. J. Colloid Interface Sci. 1987, 117, 187-192.

(70) Rübe, A.; Hause, G.; Mäder, K.; Kohlbrecher, J. Core-shell structure of Miglyol/poly(d,1-lactide)/Poloxamer nanocapsules studied by small- angle neutron scattering. J. Controlled Release 2005, 107, 244-252.

(71) Roosen-Runge, F.; Bicout, D. J.; Barrat, J. Analytical correlation functions for motion through diffusivity landscapes. J. Chem. Phys. 2016, 144, 204109. 